\title{
Growth of Beam-Plasma Instabilities in the Presence of Background Inhomogeneity
}

\author{
Mohamad Shalaby ${ }^{1,2,3}$ (), Avery E. Broderick ${ }^{1,3}$, Philip Chang ${ }^{4}$ (D), Christoph Pfrommer $^{5}$ (1), \\ Astrid Lamberts ${ }^{6}$ (iD), and Ewald Puchwein ${ }^{7}$ \\ ${ }^{1}$ Perimeter Institute for Theoretical Physics, 31 Caroline Street North, Waterloo, ON, N2L 2Y5, Canada; mshalaby@live.ca \\ ${ }^{2}$ Department of Astronomy and Astrophysics, University of Chicago, 5640 S. Ellis Avenue, Chicago, IL 60637, USA \\ ${ }^{3}$ Department of Physics and Astronomy, University of Waterloo, 200 University Avenue West, Waterloo, ON, N2L 3G1, Canada \\ ${ }^{4}$ Department of Physics, University of Wisconsin-Milwaukee, 1900 E. Kenwood Boulevard, Milwaukee, WI 53211, USA \\ ${ }^{5}$ Leibniz-Institut für Astrophysik Potsdam (AIP), An der Sternwarte 16, D-14482 Potsdam, Germany \\ ${ }^{6}$ Theoretical Astrophysics, California Institute of Technology, Pasadena, CA 91125, USA \\ ${ }^{7}$ Institute of Astronomy and Kavli Institute for Cosmology, University of Cambridge, Madingley Road, Cambridge, CB3 0HA, UK \\ Received 2018 March 8; revised 2018 April 2; accepted 2018 April 12; published 2018 May 23
}

\begin{abstract}
We explore how inhomogeneity in the background plasma number density alters the growth of electrostatic unstable wavemodes of beam-plasma systems. This is particularly interesting for blazar-driven beam-plasma instabilities, which may be suppressed by inhomogeneities in the intergalactic medium (IGM) as was recently claimed in the literature. Using high-resolution particle-in-cell simulations with the SHARP code, we show that the growth of the instability is local, i.e., regions with almost homogeneous background density will support the growth of the Langmuir waves even when they are separated by strongly inhomogeneous regions, resulting in an overall slower growth of the instability. We also show that if the background density is continuously varying, the growth rate of the instability is lower, although in all cases the system remains within the linear regime longer and the instability is not extinguished. In all cases, the beam loses approximately the same fraction of its initial kinetic energy in comparison to the uniform case at nonlinear saturation. Thus, inhomogeneities in the IGM are unlikely to suppress the growth of blazar-driven beam-plasma instabilities.
\end{abstract}

Key words: instabilities - methods: numerical - plasmas - relativistic processes - waves

\section{Introduction}

The majority of astrophysical plasmas are cold $\left(k_{B} T \ll m_{e} c^{2}\right)$ and collisionless. Plasmas that contain non-thermal (relativistic or non-relativistic) sub-populations are subject to strong beamplasma instabilities that can redistribute the energy in nonthermal populations. Of particular interest are the beam-plasma instabilities due to the propagation of the electron-positron pairs driven by the $\mathrm{TeV}$ emission of blazars in the intergalactic medium (IGM) (Broderick et al. 2012; Chang et al. 2012; Pfrommer et al. 2012; Schlickeiser et al. 2012, 2013; Vafin et al. 2018). These could lead to, e.g., a preferential heating of low-density regions of the IGM (Puchwein et al. 2012; Lamberts et al. 2015).

Linear and quasi-linear analyses of the beam-plasma instabilities assume that the background plasma is spatially uniform both when the growth rates for oblique wavemodes are computed (e.g. by Bret et al. 2010a, 2010b; Chang et al. 2016), and also when higher-order perturbative calculations are used to assess the nonlinear effects on the linear growth rates (Chang et al. 2014). However, as pointed out by Brey̌zman \& Ryutov (1971) and Miniati \& Elyiv (2013), the background inhomogeneity may be particularly problematic. In particular, it may completely suppress the effect of the instabilities for blazar-driven beam-plasma instabilities in the IGM.

A relevant characterization of the inhomogeneity in the beamplasma system is the inhomogeneity scale length along the beam direction, $\lambda_{\|}^{\text {inh }}$. This is a measure of the spatial scale over which the number density, $n$, changes significantly along the beam direction and is typically defined as $\lambda_{\|}^{\text {inh }} \equiv\left|n /(\nabla n)_{\|}\right|$. The inhomogeneity scale for the IGM at different redshifts, $z$, was computed by Miniati \& Elyiv (2013) using cosmological simulations. They find that, at mean density, $\lambda_{\|}^{\text {inh }} \sim 25,100$, and $400 \mathrm{kpc}$ for $z=3,1$, and 0 , respectively. The distance travelled by the blazar-induced pairbeams is estimated to be about $1 \mathrm{kpc}$ in one growth time, i.e., one $e$-folding (Miniati \& Elyiv 2013). Therefore, the pair-beams experience a slowly varying IGM number density.

In the presence of background plasma inhomogeneity, there are two timescales that are important for determining the effect of the inhomogeneity on the growth of the unstable wavemodes: first, the timescale for the growing wave to respond to the inhomogeneity, $\tau_{\text {inh }}$, and, second, the timescale over which the growth occurs in the linear regime, $\tau_{\mathrm{g}}$, i.e., the timescale after which nonlinear effects become important. The growth rates for the homogeneous background plasma are applicable if

$$
\tau_{\text {inh }} \gg \tau_{\mathrm{g}}
$$

When this condition is violated, i.e., $\tau_{\text {inh }} \lesssim \tau_{\mathrm{g}}$, the growth of wavemodes can still occur but at lower rates (Brě̌zman \& Ryutov 1971). However, the degree of this suppression is uncertain. Miniati \& Elyiv (2013) assumed that the unstable wavemodes are completely suppressed when the condition in Equation (1) is violated. Contrarily, Breǐzman \& Ryutov (1971) claimed that the beam loses only a negligible amount of its initial kinetic energy when this condition is violated.

In this work, we use high-resolution, one-dimensional particle-in-cell (PIC) simulations to study the effect of number density inhomogeneity. We use the SHARP code (Shalaby et al. 2017b), which provides an excellent control over typical numerical heating and energy non-conservation, while conserving the charge density (locally) and total momentum exactly. We find that even an egregious violation of the condition in Equation (1) still allows for significant growth, but at slower rates. The effect of the instability (during the linear evolution) on the beam energy loss is similar to that in the homogeneous cases. The reason is that, in presence of background plasma 
inhomogeneities, the system stays for longer times in the linear regime, saturating at similar levels.

Our one-dimensional simulations present an idealized experiment to study the effect of the background plasma inhomogeneity on the growing wavemodes in the case of blazar driven pair beams for the following reasons. First, only longitudinal wavemodeswhose growth is largely insensitive to the details of the momentum distribution of the pair-beams (Bret et al. 2010b) - are included in our simulations. Therefore, simulations that initially resolve the spectral support of the instability will be able to resolve the instabilities during the physical evolution, which typically results in increasing the width of the momentum distribution of the pairbeams. ${ }^{8}$ Second, in the linear regime, the effect of the background plasma inhomogeneity is fully decoupled from the momentum distribution of the background and pair beams. Thus, the effect of inhomogeneity on the growth of the other wavemodes (oblique and Weibel modes) is expected to be very similar to that on the longitudinal wavemodes. That is, for extreme beam parameters, correctly simulating the physical evolution is computationally tractable only for the longitudinal wavemodes, and since the effect of inhomogeneity is similar for all unstable wavemodes, our result is expected to hold when the oblique and Weibel wavemodes are correctly captured in simulations as well. However, we leave explicit demonstration of this point to future work.

Here, we focus on the case of relativistic dilute pair-beams relevant for blazar-induced pair-beams (Broderick et al. 2012). However, we note that numerical studies of the inhomogeneity effects $^{9}$ on the electrostatic wavemodes (1D) of nonrelativistic beam plasma instabilities have been previously performed in the context of solar wind plasmas (see, e.g., Krasnoselskikh et al. 2007; Voshchepynets \& Krasnoselskikh 2013; Krafft et al. 2015, 2013; Voshchepynets et al. 2015; Thurgood \& Tsiklauri 2016; Krafft \& Volokitin 2017).

This paper is organized as follows: Section 2 defines the problems with the linear perturbation analyses for the beamplasma system in the presence of inhomogeneity. In Section 3, we discuss the condition for the validity of linear growth rates in inhomogeneous plasmas and derive a general condition for growth of longitudinal wavemodes. In Section 4, we present the setup for simulations that violate this condition and discuss their numerical convergence. In Section 5 we present our simulation results in the linear and saturated, nonlinear regimes. We conclude in Section 6.

\section{Non-uniform Background Plasmas: Defining the Problem}

For a beam-plasma system with a fixed neutralizing background, we denote the phase space distribution functions of beam electrons/positrons by $f^{ \pm}$and for background electrons by $g$. The linearized (first-order) Vlasov-Maxwell equations, which describe the longitudinal evolution of a linear perturbation, are given by

$$
\partial_{t} f_{1}^{ \pm}(x, t, u)+v \partial_{x} f_{1}^{ \pm}(x, t, u) \pm \frac{e}{m_{e}} E_{1}(x, t) \partial_{u} f_{0}^{ \pm}(u)=0,
$$

\footnotetext{
8 In higher dimensions, the fastest growing wavemodes are oblique wavemodes, whose spectral width are very sensitive to the details of the beam momentum distribution (Timofeev et al. 2009; Bret et al. 2010b); thus correctly capturing the instabilities during the physical evolution is a challenging computational problem.

9 These include both simulations and numerical solutions of the Zakharov equations (approximate nonlinear evolution equations).
}

$$
\begin{aligned}
\partial_{t} g_{1}(x, t, u)+v \partial_{x} g_{1}(x, t, u)-\frac{e}{m_{e}} E_{1}(x, t) \partial_{u} g_{0}(x, u)=0 \\
\begin{aligned}
\partial_{x} E_{1}(x, t)= & \frac{e}{\epsilon_{0}} \int\left[f_{1}^{+}(x, t, u)\right. \\
& \left.-f_{1}^{-}(x, t, u)-g_{1}(x, t, u)\right] d u
\end{aligned}
\end{aligned}
$$

where $e$ and $m_{e}$ are the elementary charge and mass of electrons, $v$ is the velocity in phase space, $u=\gamma v$ with $\gamma=1 / \sqrt{1-v^{2}}, f_{0}^{ \pm}$and $f_{1}^{ \pm}$are the equilibrium and the firstorder perturbation of the phase space distribution function of pair-beam plasma particles, respectively, $g_{0}$ and $g_{1}$ are the corresponding quantities of the background electron plasma, respectively, and $E_{1}$ is the first-order perturbation in the electric field.

Due to the inhomogeneously distributed background electrons, the equilibrium distribution function $g$ depends on the position $x$. To solve these equations as an initial-value problem (using the Landau procedure), one takes the Fourier/ Laplace transform for $x / t$ and assumes initial perturbations for the pair-beam plasmas $f_{\text {ini }}^{ \pm}=f_{1}^{ \pm}(x, u, t=0)$ and for the electron background plasma $g_{\text {ini }}=g_{1}(x, u, t=0)$ (Landau 1946; Nicholson 1983; Boyd \& Sanderson 2003). We get the following equations:

$$
\begin{gathered}
i k E_{1}(k, \omega)=\frac{e}{\epsilon_{0}} \int\left[f_{1}^{+}-f_{1}^{-}-g_{1}\right] d u, \\
f_{\text {ini }}^{ \pm}(k, u)=-i(\omega-k v) f_{1}^{ \pm}(k, \omega, u) \pm \frac{e \partial_{u} f_{0}^{ \pm}(u)}{m_{e}} E_{1}(k, \omega), \\
g_{\text {ini }}(k, u)=-i(\omega-k v) g_{1}(k, \omega, u) \\
-\frac{e}{m_{e}} \int d k^{\prime} E_{1}\left(k-k^{\prime}, \omega\right) \partial_{u} g_{0}\left(k^{\prime}, u\right),
\end{gathered}
$$

where, $f_{\text {ini }}^{ \pm}(k, u)$ and $g_{\text {ini }}(k, u)$ are the Fourier transform of $f_{\text {ini }}^{ \pm}(x, u)$ and $g_{\text {ini }}(x, u)$, respectively. Therefore,

$$
\begin{array}{r}
{\left[k+\frac{e^{2}}{m_{e} \epsilon_{0}} \int \frac{d u}{\omega-k v} \partial_{u}\left(f_{0}^{+}+f_{0}^{-}\right)\right] E_{1}(k, \omega)} \\
+\frac{e^{2}}{m_{e} \epsilon_{0}} \iint d k^{\prime} d u \frac{\partial_{u} g_{0}\left(k^{\prime}, u\right)}{\omega-k v} E_{1}\left(k-k^{\prime}, \omega\right) \\
=\frac{e}{\epsilon_{0}} \int \frac{d u}{\omega-k v}\left[f_{\text {ini }}^{+}-f_{\text {ini }}^{-}-g_{\text {ini }}\right]
\end{array}
$$

The convolution in Equation (8) implies coupling between all Fourier modes of $E_{1}(k, \omega)$ with all Fourier modes in the background plasma inhomogeneity, i.e., a nonlinear coupling of the modes to the structure in the background. This is a direct consequence of the fact that, in general, the normal wavemodes of an inhomogeneous plasma are not Fourier modes. In other words, the Fourier modes do not describe linearly independent solutions to the linearized equations.

\section{Applicability of Homogeneous Plasma Linear Growth Rates in the Presence of Inhomogeneity}

First, we follow the empirical discussion in Breǐzman \& Ryutov (1971) and Miniati \& Elyiv (2013) and quantify the timescales that determine whether the homogeneous plasma 
linear growth rates are applicable. Then, we derive the applicability condition for uniform plasma growth rates of longitudinal unstable wavemodes in the presence of inhomogeneity.

\subsection{General Discussion}

To simplify, we consider only inhomogeneity along the pairbeam direction. The timescale on which inhomogeneity affects the growing wavemode can be found using the geometric optics approximation:

$$
\frac{d k}{d t}=\frac{d \omega}{d x} \sim \frac{\omega_{g}}{2 \lambda_{\|}^{\text {inh }}}
$$

where $\lambda_{\|}^{\text {inh }}$ is the inhomogeneity scale length in the pair-beam direction and $\omega_{g}$ is the background plasma frequency at mean density. Therefore, if the width of unstable oblique wavemodes along the beam direction is $\Delta k_{\|}$, then the inhomogeneity timescale is given by

$$
\tau_{\text {inh }} \equiv \frac{\Delta k_{\|}}{|d k / d t|} \sim \frac{\Delta k_{\|} 2 \lambda_{\|}^{\text {inh }}}{\omega_{g}} .
$$

The timescale associated with the fastest oblique wavemode linear growth is given by

$$
\tau_{\mathrm{obl}} \equiv \frac{\Lambda^{\mathrm{obl}}}{\Gamma^{\mathrm{obl}}},
$$

where $\Lambda^{\text {obl }}$ is the number of $e$-foldings for oblique wavemodes before the nonlinear effects become important and $\Gamma^{\mathrm{obl}}$ is the maximum growth rate of oblique wavemodes. Therefore, the condition for the validity of linear homogeneous growth rate in the presence of background inhomogeneity, $\tau_{\text {inh }} \gg \tau_{\text {obl }}$, corresponds to

$$
\lambda_{\|}^{\mathrm{inh}} \gg \lambda_{\min } \equiv \frac{\Lambda^{\mathrm{obl}}}{2} \frac{\omega_{g}}{\Gamma^{\mathrm{obl}}} \frac{1}{\Delta k_{\|}} .
$$

Previously, Miniati \& Elyiv (2013) argued that the violation of Equation (12) results in a severe suppression of the linear growth rate. We now test this suppression using a series of $1 \mathrm{D}$ numerical simulations in which the condition is violated. We find that the maximum growth rate is slower than predicted for the homogeneous case but the unstable wavemodes still grow and the pair beam energy loss (in the linear growth) is approximately the same as that of the homogeneous plasma case. ${ }^{10}$

\subsection{Longitudinal Unstable Wavemodes}

Here, we derive a condition for the validity of the electrostatic growth rates (in the cold limit) using the correct spectral width found by Shalaby et al. (2017a). For a uniform background, the dispersion relation for Langmuir (longitudinal) waves, in the cold limit, is given by (Faǐnberg et al. 1969)

$$
1-\frac{1}{\hat{\omega}^{2}}-\frac{\alpha / \gamma_{b}^{3}}{(\hat{\omega}-\hat{k})^{2}}=0,
$$

\footnotetext{
${ }^{10}$ We note that the spectral width for oblique unstable wavemodes, $\Delta k_{\|}$, used in (Brě̌zman \& Ryutov 1971; Miniati \& Elyiv 2013) reduces to $\Delta k_{\|}=0$ in the cold limit. However, when the cold-limit dispersion relation is solved a finite spectral width of wavemodes growing with rates comparable to the maximum growth rates exits (see, e.g., Bret et al. 2010a).
}

Table 1

Electrostatic Beam-Plasma Instability Simulations with $\alpha=0.002, \gamma_{b}=100$

\begin{tabular}{llcccc}
\hline \hline Simulation & $L_{c}{ }^{\mathrm{a}}$ & $\Gamma^{\mathrm{sim}} / \Gamma_{\max }^{\mathrm{th}}{ }^{\mathrm{b}}$ & $N_{\mathrm{pc}}{ }^{\mathrm{c}}$ & $\lambda_{\|}^{\mathrm{inh}}$ & $E_{r} / E_{b, 0}{ }^{\mathrm{e}}$ \\
\hline "Uniform" & 263.9 & 0.883 & 2706.4 & $\infty$ & $0.0017 \%$ \\
"Bump" & 861 & 0.75 & 3491.3 & $\sim 80 \mathrm{c} / \omega_{p}$ & $0.0013 \%$ \\
"Cosine" & 128 & 0.20 & 3914.0 & $\sim 200 c / \omega_{p}$ & $0.0012 \%$ \\
"Exponential" & 125 & 0.10 & 4008.0 & $\sim 62.5 c / \omega_{p}$ & $0.0005 \%$
\end{tabular}

${ }^{a}$ The box size, $L$, in units of skin depth, i.e., $L_{c}=L \omega_{p} / c$, where $\omega_{p}$ is the plasma frequency associated with all plasma particles: beam and background particles.

${ }^{\mathrm{b}}$ The maximum growth rate found in simulations $\Gamma^{\mathrm{sim}}$ normalized to the maximum growth rate predicted theoretically for a uniform plasma, $\Gamma_{\max }^{\text {th }}=8.647 \times 10^{-4} \omega_{p}$, found by solving the dispersion relation in Equation (13).

${ }^{c}$ Total number of macro-particles (background electrons and beam electrons and positrons) divided by the total number of computational cells.

${ }^{d}$ The inhomogeneity scale length; the scale length on which the background plasma number density changes significantly. To obtain the degree of violation for the condition of the validity of homogeneous growth rates $\left(\lambda_{\|}^{\mathrm{inh}} \ll \lambda_{\min }\right)$, this should be compared to $\lambda_{\min } \sim 8.38 \times 10^{5} c / \omega_{p}$, i.e., this condition is violated by about three order of magnitudes in all non-uniform simulations.

${ }^{\mathrm{e}}$ Maximum energy error in simulations normalized to the initial energy of the pair-beam particles.

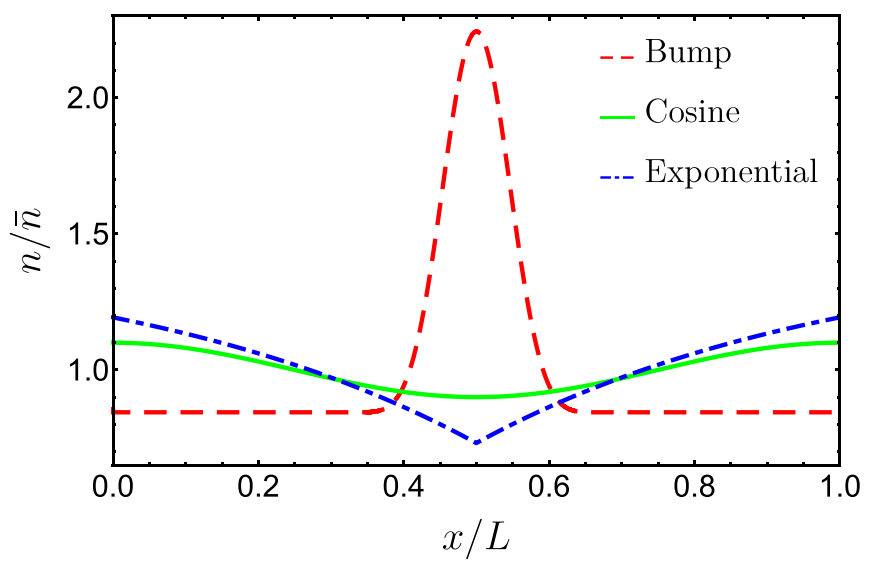

Figure 1. Initial background plasma number density (electrons and fixed ions) for the various inhomogeneous simulations. All simulations are 1D with a computational domain of length $L$. For each, the number density $n$ is normalized by the average number density $\bar{n}$.

where $\hat{\omega}=\omega / \omega_{g}, \hat{k}=k v_{b} / \omega_{g}, \quad v_{b}$ is the beam velocity, $\alpha=n_{b} / n_{g}, \omega_{g}=\sqrt{n_{g} e^{2} / m_{e} \epsilon_{0}}$ is the background plasma frequency, and $n_{g}$ and $n_{b}$ are the number densities of background and beam plasma, respectively. The dispersion relation in the linear regime, Equation (13), implies instability (exponential growth) for all wavemodes with wavelengths (Shalaby et al. 2017a)

$$
\lambda \geqslant \frac{2 \pi v_{b}}{\omega_{g}}\left(1+\frac{\alpha^{1 / 2}}{\gamma_{b}}\right)^{-3 / 2} \Rightarrow k \leqslant\left(1+\frac{\alpha^{1 / 2}}{\gamma_{b}}\right)^{3 / 2} \frac{\omega_{g}}{v_{b}} .
$$

The spectral width, $\Delta k_{\|}$, of these unstable wavemodes along the beam direction is given by (using Equation (10) of 


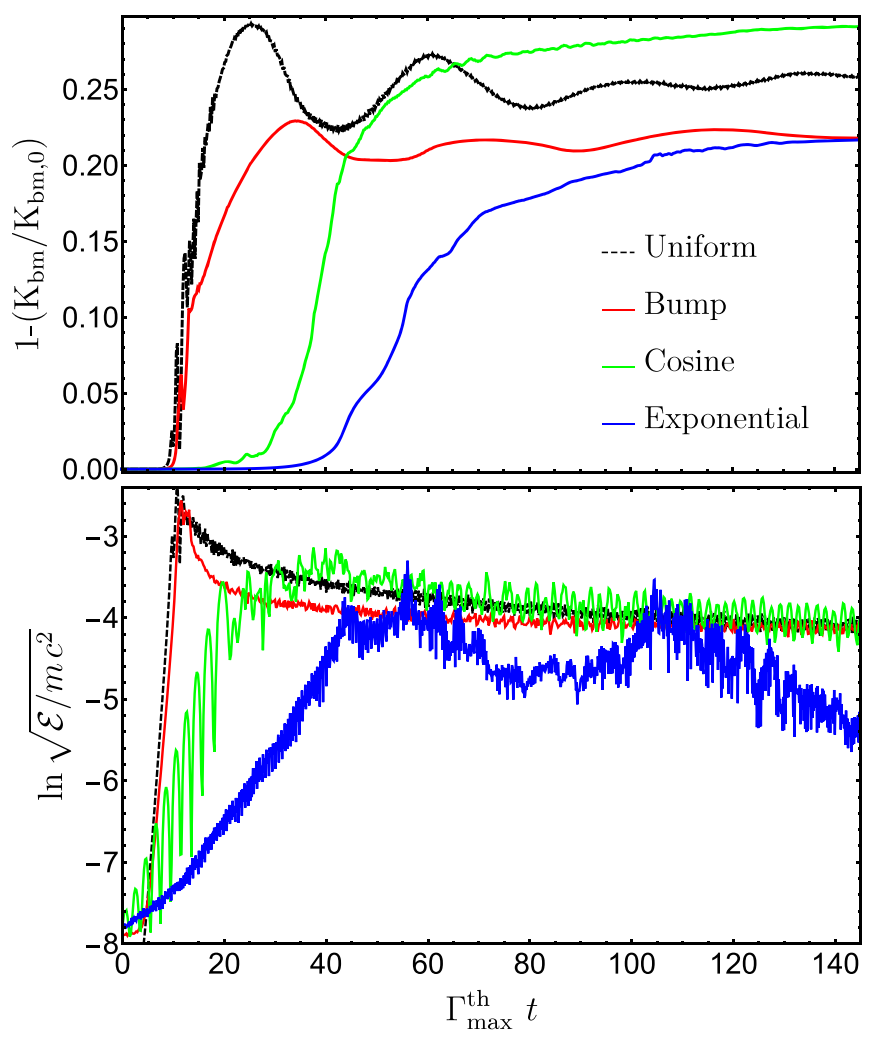

Figure 2. Top: evolution of the fractional energy loss of beam particles in different simulations with inhomogeneous and homogeneous background plasmas. Bottom: evolution of the total potential energy per computational particle, $\mathcal{E}$, normalized to the rest mass energy of a computational particle, $m c^{2}$. Since the growth in all simulations starts from the Poisson noise, the times are shifted in different simulations (by a maximum of $\Gamma_{\max }^{\text {th }} t=7$, depending on resolution) to allow a direct comparison of the exponential growth rates of the potential energy.

Shalaby et al. 2017a)

$$
\Delta k_{\|} \approx \Delta k_{1 / 2}=\frac{2 \pi}{1.15008}\left(\frac{\alpha}{\gamma_{b}^{3}}\right)^{1 / 3} \omega_{g} / c,
$$

where $\Delta k_{1 / 2}$ is the full width at half maximum of the unstable wavemodes, i.e., it is the $k$-space width of unstable wavemodes that grow with rates $\geqslant 0.5$ of the maximum growth rate. The maximum growth rate for these wavemodes is given by (Bret et al. 2010c)

$$
\Gamma^{\mathrm{L}} \sim \frac{\sqrt{3}}{2^{4 / 3}}\left(\frac{\alpha}{\gamma_{b}^{3}}\right)^{1 / 3} \omega_{g}
$$

Therefore, for the longitudinal unstable wavemodes

$$
\begin{aligned}
\lambda_{\min } & =\frac{\Lambda^{\mathrm{L}}}{2} \frac{2^{4 / 3}}{\sqrt{3}} \frac{1.15008}{2 \pi}\left(\frac{\alpha}{\gamma_{b}^{3}}\right)^{-2 / 3} \frac{c}{\omega_{g}} \\
& =1.33\left(\frac{\Lambda^{\mathrm{L}}}{10}\right)\left(\frac{\alpha}{\gamma_{b}^{3}}\right)^{-2 / 3} c / \omega_{g} .
\end{aligned}
$$

\section{Numerical Simulations: Setup and Convergence}

Here, we present a number of 1D simulations with inhomogeneity that strongly violate the condition $\lambda_{\|}^{\text {inh }} \gg \lambda_{\text {min }}$, with $\lambda_{\text {min }}$ given in Equation (17). The growth of unstable wavemodes and energy loss from the pair-beams are contrasted with the corresponding results of a uniform background plasma simulation. While we find that the growth rates are reduced modestly in presence of background plasma inhomogeneity, the pair-beam energy loss is found to be similar in all simulations.

\subsection{Simulation Setup}

For our numerical simulations, we use SHARP-1D (Shalaby et al. 2017b) with fifth-order interpolation, $W^{5}$, to improve the conservation of energy in simulations while conserving the total momentum exactly. Using SHARP with $W^{5}$ is essential to avoid the excessive numerical heating typical in most available PIC codes. Importantly it eliminates numerical heating for long-time simulations (millions of $\omega_{p}^{-1}$, see Shalaby et al. 2017 for illustration).

In all simulations, we resolve the plasma skin depth, $c / \omega_{p}$, by 20 cells, i.e., the cell size is $\Delta x=0.05 c / \omega_{p}$, and use a time step that satisfies the Courant-Friedrichs-Lewy stability condition; we used $c \Delta t / \Delta x=0.4$. The momentum distributions of the beam and background plasmas (in their individual comoving frames) are initialized using a thermal distribution with normalized temperatures $\theta_{g}=\theta_{b}=4 \times 10^{-3}$, where $\theta=k_{B} T / m_{e} c^{2}$. In all cases periodic boundary conditions are applied, and other simulation parameters are laid out in Table 1.

We assume a background plasma (electrons and immobile ions) that is spatially inhomogeneous, but charge neutral, in all simulations. We also assume a spatially uniform pair-beam plasma (electrons and positrons) that moves with a Lorentz factor of $\gamma_{b}=100$, and a beam-to-background ratio of $\alpha=n_{b} / n_{g}=0.002$, where $n_{b}$ and $n_{g}$ are the number density of beam particles and background electrons, respectively. ${ }^{11}$ As a result, the pair beam is highly relativistic but energetically subdominant, similar to those anticipated in the IGM. For all simulations,

$$
\lambda_{\min } \sim 8.38 \times 10^{5} c / \omega_{p}
$$

where we $\operatorname{set}^{12} \Lambda^{\mathrm{L}}=10$. Since, in all inhomogeneous simulations, the inhomogeneity scale length $\lambda_{\|}^{\text {inh }} \lesssim 200 c / \omega_{p}$, the condition in Equation (1) is violated by more than three orders of magnitude in all cases.

We consider three cases which fit within two classes of background inhomogeneities, distinguished by the extent of the background variations. The first of these is the "Bump" simulation; a simulation with a central Gaussian bump in the background density. The periodic simulation domain is divided into three parts; in the first and last part the number density is uniform, while in the middle part the number density follows a Gaussian distribution with standard deviation, $\sigma=(L / 20)$; red curve in Figure 1).

Within the second class, we present two simulations with a continuously varying background density. In the first, the background plasma number density is varying as a cosine across the box (with amplitude, $A=0.1$ ); green curve in Figure 1. We call this the "Cosine" simulation. In the second,

\footnotetext{
11 Note: here $n_{b}$ is the number density of all beam particles (both electrons and positrons).

${ }^{12}$ Typically, it is assumed that $\Lambda^{\mathrm{L}} \sim 30$ (Huba 2013; Miniati \& Elyiv 2013). However, we set $\Lambda^{\mathrm{L}}=10$, since we observe from the second panel of Figure 2 that it is of order 10. Setting it to higher values implies higher values for $\lambda_{\min }$, and thus a stronger violation of the condition in Equation (12) by the same factor.
} 


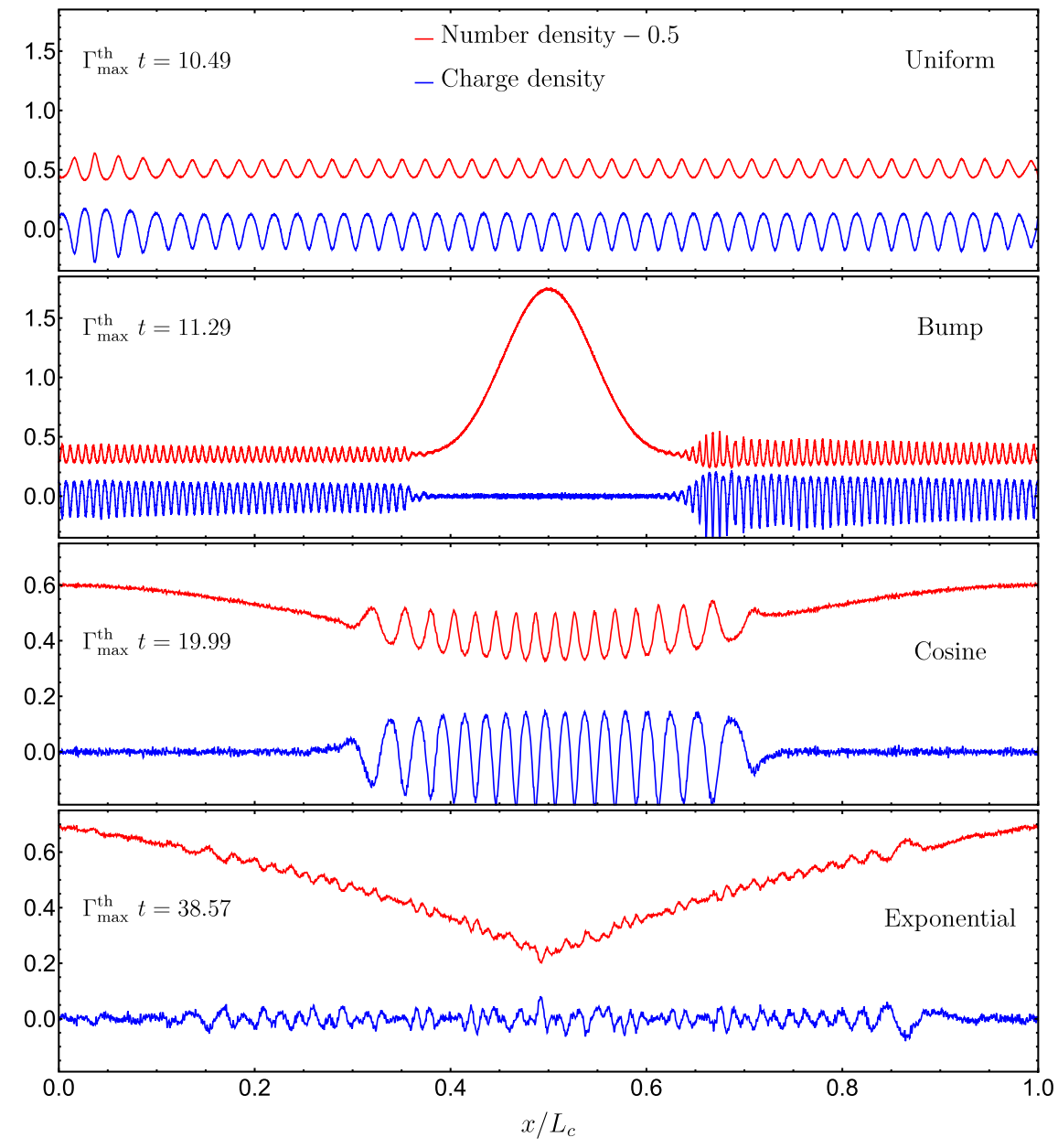

Figure 3. Instantaneous charge density (red) and number density (blue) close to the end of the linear evolution for the different inhomogeneous and homogeneous background plasma simulations. In all cases, unstable wavemodes are excited despite variations in the background density.

the variation is much faster; the number density at the edges of the computational domain is about 1.2 of the average number density, $\bar{n}$, and drops exponentially fast until it reaches $0.73 \bar{n}$ in the middle of the computational domain and then increases exponentially fast afterwards to reach $1.2 \bar{n}$ at the other edge of the computational domain (see blue curve in Figure 1). We call this the "Exponential" simulation. More precisely, the number density in the "Exponential" simulation is given by

$$
\frac{n(x / L)}{\bar{n}}=\frac{2 e}{1+e}-\frac{e^{1-2|x / L-0.5|}}{1+e} .
$$

Since the theoretically expected maximum growth rate is $\Gamma_{\max }^{\text {th }}=8.647 \times 10^{-4} \omega_{p}$, during one growth time, beam particles travelling with $v_{b} \sim c$ will travel a distance $\sim 1156 \mathrm{c} / \omega$. That is, during one growth time, the beam particles will travel distances larger than the box size in all simulations. Since the growth rates in nonuniform simulations are lower than $\Gamma_{\max }^{\text {th }}$ (see Table 1), the distance travelled by the beam, during one growth time in the simulation is even larger than $1156 c / \omega_{p}$.

\subsection{Simulation Convergence and Performance}

Convergence of numerical simulations is an essential way to avoid confusing the evolution of different numerical errors with physical evolution. In Shalaby et al. (2017b), we demonstrated that the typical method of checking the convergence in PIC simulations is misleading: increasing the number of particles per cell $\left(N_{\text {pc }}\right)$ and decreasing the cell sizes $(\Delta x)$ independently was shown to lead to a plateau in the numerical errors. Thus changing these parameters independently does not imply convergence as typically claimed. The correct convergence was shown to be only possible when both of these resolution criteria are improved simultaneously.

We follow this approach to check the convergence for all inhomogeneous simulations presented here, and present the results from the highest resolved simulations. For all inhomogeneous simulations presented here, the beam energy evolution and the grid potential energy evolution are very similar to those in simulations with resolution lower by a factor of 2 , i.e., with $0.5 N_{\mathrm{pc}}$ and $2 \Delta x$.

For uniform simulations, the uniformity coupled with the periodicity on the physical domain imply a minimal spectral width within which wavemodes are not resolved in simulations. This means that there is another resolution criterion (box size $L$ ) that should be also improved (independently or simultaneously with other resolution criteria) in order to resolve the narrow spectral width of the unstable wavemodes of relativistic and dilute pair-beam instabilities (Shalaby et al. 2017a). Thus, for uniform simulations, we perform them with resolution increased by factors of 2 and 4 , i.e., increase $L$ and $N_{\mathrm{pc}}$ and decrease $\Delta x$ by such a factor simultaneously. In all simulations we obtain very similar 

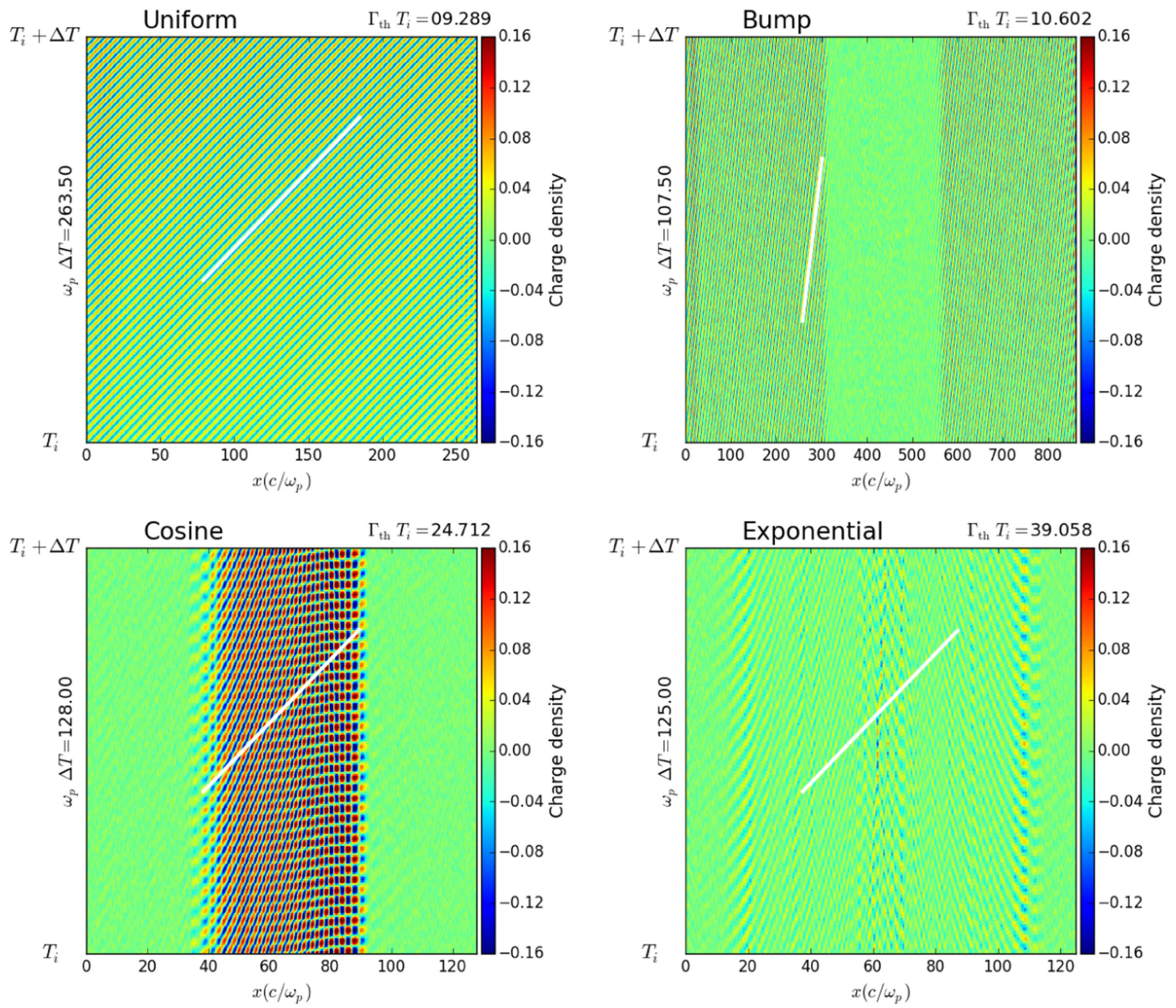

Figure 4. Growth and evolution of the charge density for different simulations close to the end of the linear regime evolution. The charge density is color coded as a function of position in the simulation box and and over a time period $T_{i}<t<T_{i}+\Delta T$ as indicated in the labels in each panel. In all panels, wavemodes that travel along white lines are travelling with the speed of light along the direction of the beam $\left(\sim v_{b}\right)$. Checkerboard patterns indicate standing waves, and thus the presence of both forward- and backward-propagating wavemodes.

pair-beam energy evolution and grid potential energy evolution; we present results of the lower resolution uniform simulation here to facilitate comparisons with inhomogeneous simulations. ${ }^{13}$

As pointed out above, the use of higher-order interpolation functions greatly reduces the numerical heating typical in PIC simulations. The maximum energy errors (normalized to the initial beam energy) in our simulations are always below $0.002 \%$, i.e., the energy error is less than $2 \times 10^{-5}$ of the initial beam energy, and less than $0.1 \%$ of the background thermal energy (see Table 1 for details).

\section{Simulation Results}

\subsection{Linear Regime Evolution}

In Figure 2, we show the evolution of the fractional beam energy loss (top) and the electric potential energy (bottom). The

\footnotetext{
${ }^{13}$ A uniform simulation with a box size smaller by a factor of 2 compared to the uniform simulation presented here results in very different energy evolution and lower energy saturation level $(\sim 14 \%)$ and also results in a slower growth of the potential energy on the simulation grid.
}

potential energies grow at smaller rates of about $0.75,0.2$, and 0.1 of the maximum growth rate for uniform plasmas for "Bump," "Cosine," and "Exponential" simulations, respectively. Figure 3 shows the growth of unstable plasma wavemodes even when the condition is severely violated.

In all simulations, the level at which the beam energy loss stops occurring with rates comparable to the linear growth rates is the same, i.e., about $20 \%-26 \%$ energy loss. This is similar to the level of saturation we obtained from a uniform background simulation with the same beam parameters. The growth rate of the uniform simulation is 0.883 of the maximum growth rate predicted from theory. This is in perfect agreement with the maximum growth rate for wavemodes allowed to grow in such a simulation box with periodic boundary conditions; see Shalaby et al. (2017a) for more details.

For the "Bump" simulation, the inhomogeneity scale length is between those of the "Cosine" and "Exponential" simulations. However, since $2 / 3$ of the box in the "Bump" simulation is uniform, the effective growth rate is higher than in the cases 

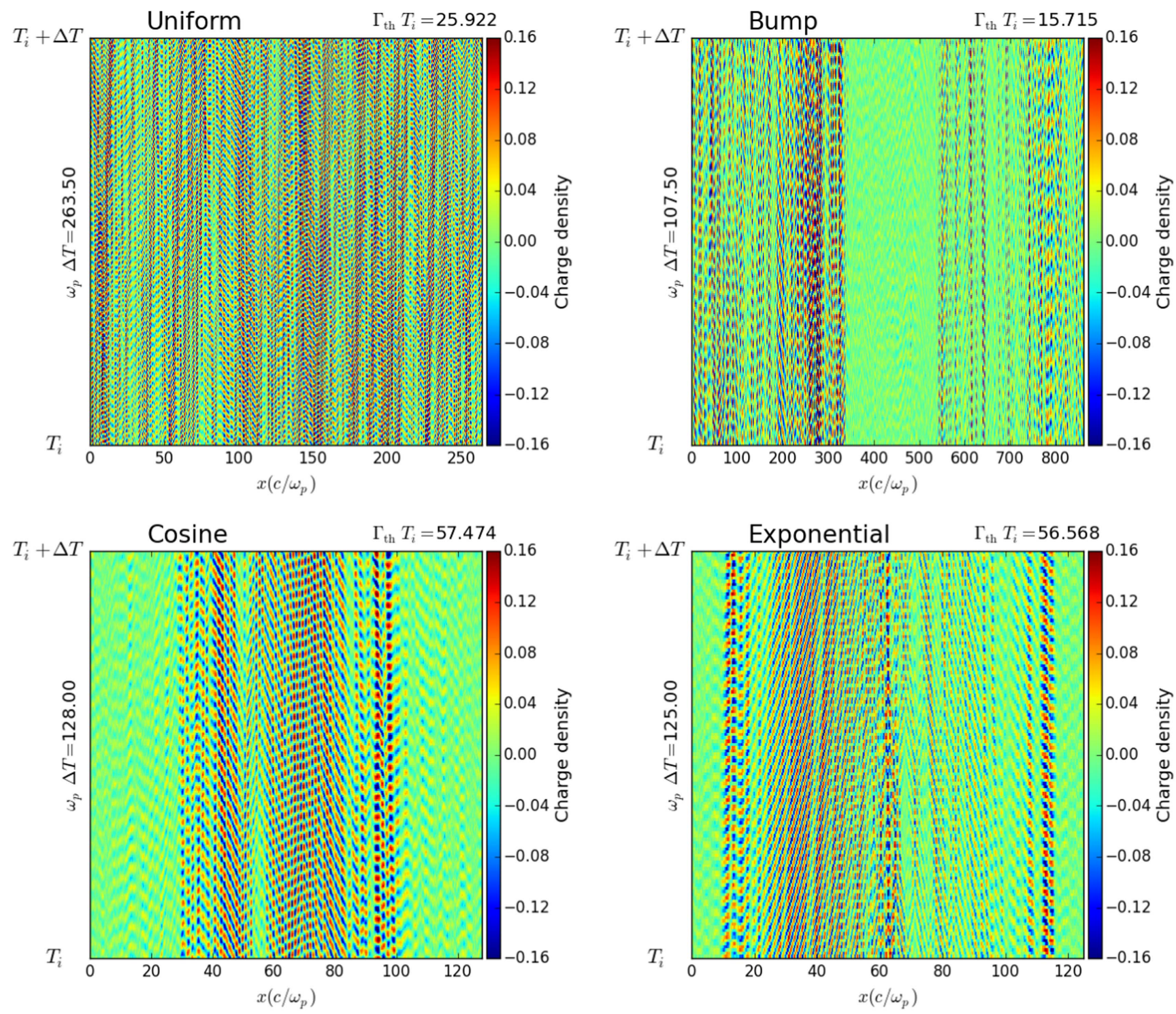

Figure 5. Saturated state of the charge density in different simulations in the nonlinear regime. The charge density is color coded as a function of position in the simulation box and and over a time period $T_{i}<t<T_{i}+\Delta T$ as indicated in the labels in each panel.

where the background number density is varying throughout the simulation domain. As seen in the second panel of Figure 3, displaying the "Bump" simulation, the growth of plasma waves is localized in the uniform regions despite the fact that the group velocity of the growing wavemodes is equal to the beam velocity $(\sim c)$. This implies that the initially excited forwardpropagating wavemodes reflect at high-density regions.

Figure 4 shows further evidence that growing modes reflect as they propagate toward high-density regions, which shows a representative period for the charge density during the linear regime evolution for each simulation. For the "Uniform" simulations, the fastest-growing wavemode is a propagating wave with group velocity equal to the beam velocity $(\sim c)$. This agrees with the linear regime prediction derived by solving the dispersion relation in Equation (13). A similar pattern can be seen in the uniform regions of the "Bump" simulation.

On the other hand, the linear regime evolution of charge density in the "Cosine" simulation shows clear wave reflections at the higher-density regions, indicated by the checkerboard patterns. This occurs at $x \sim 90 c / \omega_{p}$, i.e., when the number density is about three times larger than that in the lowest density region (i.e., at $x=64 c / \omega_{p}$ ). A similar pattern (wave reflections during the linear regime evolution) is also seen in the "Exponential" simulation.

\subsection{Saturated Nonlinear Regime}

Analytically, it is hard to identify the reason(s) for the similar level of beam energy loss that is approximately achieved in simulations with both homogeneous and inhomogeneous background plasmas. This is the case, despite the modestly lower growth rates (during the linear regime) of inhomogeneous plasma simulations. Therefore, in this section we present different representations of the nonlinearly saturated state, i.e., after the end of linear growth. The saturated state looks very similar in all simulations, which is consistent with the similar energy loss. However, the evolution to achieve this state is different in different simulations.

In Figure 5, we show the nonlinear saturated state for the charge density in all simulations. In the nonlinear regime, the 

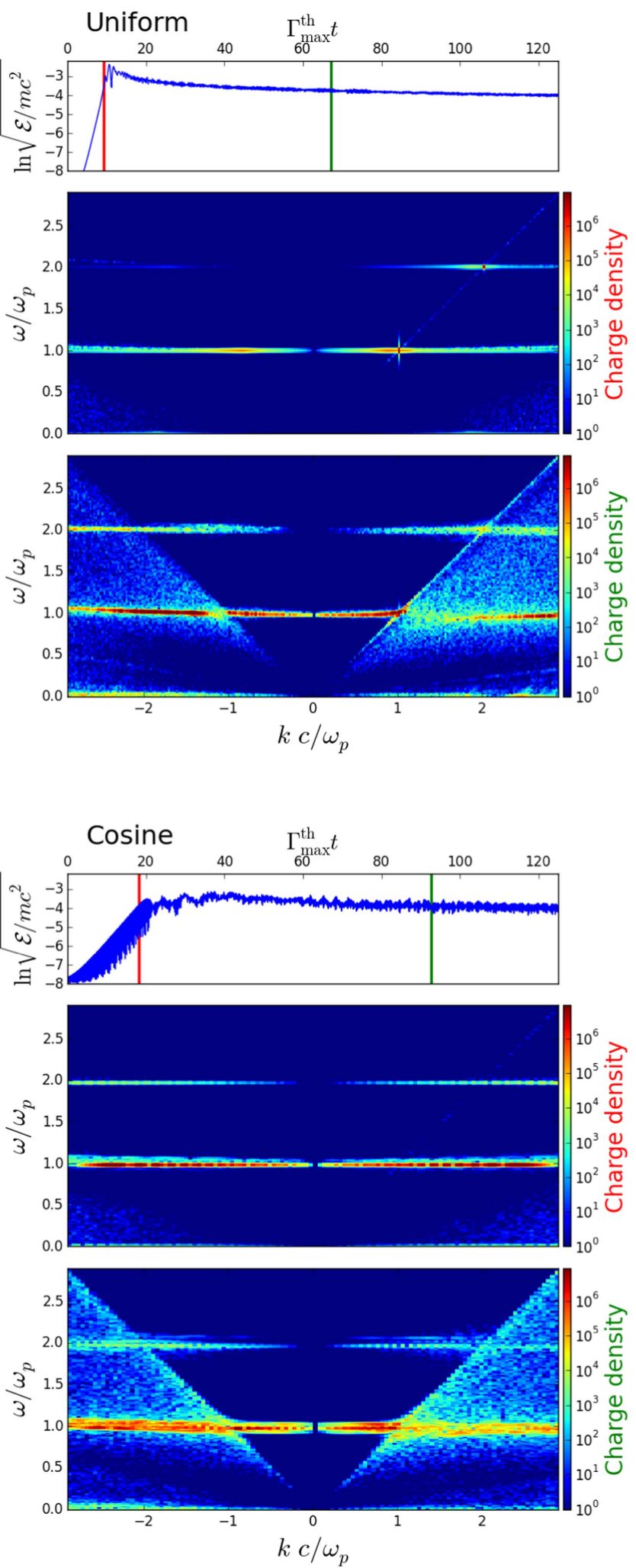
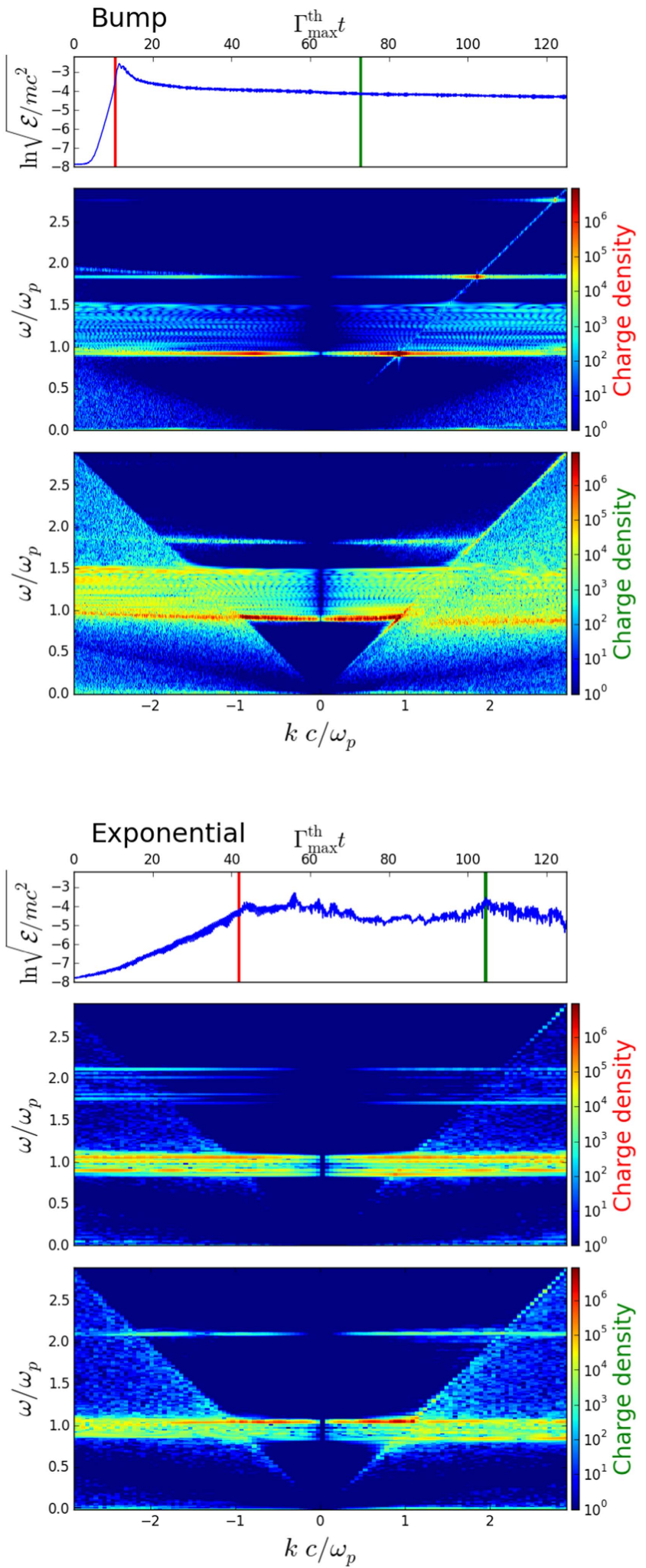

Figure 6. Distribution of power (i.e., the square of the absolute value of the charge density 2D Fourier transform) of the excited beam-plasma wavemodes in all simulations. For each simulation we show the evolution of the normalized potential energy from the linear to the saturated nonlinear regime (top panel). The middle panel shows the distribution of power at the end of the linear evolution, as indicated by the red vertical line in the top panel. The bottom panel shows the distribution of power in the saturated nonlinear regime, as indicated by the green vertical line in the top panel.

charge density evolution is complicated and clearly differs from the linear regime due to nonlinear interactions between all linearly unstable, growing wavemodes. Figure 5 shows that the saturated state in all simulations is similar, suggesting that the saturation in uniform and non-uniform simulations occurs via similar physical mechanism(s) despite the difference in their evolution in the linear regime. This is also consistent with the similar degrees of energy loss from the pair-beams in all 
simulations at this state. Characterizing the physical mechanism(s) and understanding the reason(s) why they do not depend on the background plasma inhomogeneity are left for future work.

In Figure 6, we present the distribution of power (i.e., the square of the absolute value of the charge density 2D Fourier transform in $(\omega, k)$-space) of different simulations at two times. First, close to the end of the linear evolution of the grid potential energy (red) and, second, in the nonlinear, saturated regime (green). In the linear regime, the evolution of the homogeneous and inhomogeneous simulations are very different. The presence of inhomogeneities increase the support of the unstable region in the $(\omega, k)$-space during the linear regime.

In the linear regime, the power in both forward- and backward-propagating wavemodes in the inhomogeneous simulations is a clear sign of the reflection of forwardpropagating wavemodes that are initially excited locally due to the propagation of pair beams. In the nonlinear regime, all simulations evolve to a similar physical state, where the power in linearly excited wavemodes cascades to lower $k$ (longer wavelengths). However, in the inhomogeneous simulations the support for growing wavemodes, in $(\omega, k)$-space, is larger than that in the uniform simulations during the linear evolution and also when the nonlinear saturated state is achieved.

\section{Conclusions}

Following previous work, we derive the condition where the linear growth rates that assume a uniform background might be not applicable in the presence of background inhomogeneity (Equation (12)). While previous work by Miniati \& Elyiv (2013) assumes that violation of this condition results in complete suppression of these beam-plasma instabilities, we demonstrated using high-resolution numerical simulations that, in fact, the instability growth rates are reduced by only a factor of a few. Moreover, the nonlinear saturation level of the instability measured in terms of the initial beam kinetic energy is broadly similar to that in the uniform background plasma case.

In the present work, the insensitivity of the level of energy loss by the pair-beam to the background plasma inhomogeneity is explicitly demonstrated only for longitudinal unstable wavemodes. We leave demonstrating this for oblique and perpendicular unstable wavemodes to future studies. However, since the effect of the inhomogeneity is expected to be similar for other unstable wavemodes, our finding is likely to hold for these cases as well.

The parameters of the beam-plasma system in the IGM are extreme $\left(\alpha=10^{-15}\right.$, and $\left.\gamma_{b} \sim 10^{6}\right)$ (Broderick et al. 2012), which result in extreme separation of scales, e.g., the growth timescales are about nine orders of magnitude longer compared to the plasma timescale $\left(\omega_{p}^{-1}\right)$. This makes simulating such beam-plasma systems with realistic parameters intractable. Since the parameters in our simulations are, however, in the correct asymptotic regime, i.e., the pair-beams are subdominant in both number and energy densities compared to the background plasma ( $\alpha=0.002$, and $\alpha \gamma_{b}=0.2$ ), we expect our conclusions here to be directly applicable for the beamplasma system of the IGM.

This suggests that blazar-driven beams will remain subject to virulent linear instabilities even in the presence of realistic levels of the inhomogeneity in the IGM. The lack of the suppression of the plasma instabilities due to background plasma inhomogeneities is consistent with the lack of $\gamma$-ray halos expected around TeV blazars if plasma instabilities were suppressed (Broderick et al. 2016; Tiede et al. 2017a, 2017b).

\section{Supplementary Material}

Links for $(x-t)$ density evolution movies: Cosine, Uniform, Bump, Exponential.

M.S. and A.E.B. received financial support from the Perimeter Institute for Theoretical Physics and the Natural Sciences and Engineering Research Council of Canada through a Discovery Grant. Research at Perimeter Institute is supported by the Government of Canada through Industry Canada and by the Province of Ontario through the Ministry of Research and Innovation. P.C. gratefully acknowledges support from NSF grant AST-1255469. C.P. acknowledges support by the European Research Council under ERC-CoG grant CRAGSMAN-646955. A.L. receives financial support from an Alfred P. Sloan Research Fellowship, NASA ATP Grant NNX14AH35G, and NSF Collaborative Research Grant 411920 and CAREER grant 1455342. E.P. acknowledges support by the Kavli Foundation.

\section{ORCID iDs}

Mohamad Shalaby (ib https://orcid.org/0000-0001-9625-5929 Philip Chang (i) https://orcid.org/0000-0002-2137-2837 Christoph Pfrommer (i) https://orcid.org/0000-00027275-3998

Astrid Lamberts (iD https://orcid.org/0000-0001-8740-0127

\section{References}

Boyd, T. J. M., \& Sanderson, J. J. 2003, The Physics of Plasmas (Cambridge: Cambridge Univ. Press)

Breǐzman, B. N., \& Ryutov, D. D. 1971, JETP, 33, 220

Bret, A., Gremillet, L., \& Bénisti, D. 2010a, PhRvE, 81, 036402

Bret, A., Gremillet, L., \& Dieckmann, M. E. 2010b, PhPl, 17, 120501 Bret, A., Gremillet, L., \& Dieckmann, M. E. 2010c, PhPl, 17, 120501 Broderick, A. E., Chang, P., \& Pfrommer, C. 2012, ApJ, 752, 22 Broderick, A. E., Tiede, P., Shalaby, M., et al. 2016, ApJ, 832, 109 Chang, P., Broderick, A. E., \& Pfrommer, C. 2012, ApJ, 752, 23

Chang, P., Broderick, A. E., Pfrommer, C., et al. 2014, ApJ, 797, 110 Chang, P., Broderick, A. E., Pfrommer, C., et al. 2016, ApJ, 833, 118 Faǐnberg, Y. B., Shapiro, V. D., \& Shevchenko, V. I. 1969, JETP, 30, 528 Huba, J. D. 2013, NRL PLASMA FORMULARY Supported by The Office of Naval Research (Washington, DC: Naval Research Laboratory), https:// www.nrl.navy.mil/ppd/content/nrl-plasma-formulary

Krafft, C., \& Volokitin, A. 2017, JPIPh, 83, 705830201

Krafft, C., Volokitin, A. S., \& Krasnoselskikh, V. V. 2013, ApJ, 778, 111 Krafft, C., Volokitin, A. S., \& Krasnoselskikh, V. V. 2015, ApJ, 809, 176 Krasnoselskikh, V. V., Lobzin, V. V., Musatenko, K., et al. 2007, JGRA, 112, A10109

Lamberts, A., Chang, P., Pfrommer, C., et al. 2015, ApJ, 811, 19

Landau, L. D. 1946, J. Phys. (USSR), 10, 25

Miniati, F., \& Elyiv, A. 2013, ApJ, 770, 54

Nicholson, D. R. 1983, Introduction to Plasma Theory (New York: Wiley)

Pfrommer, C., Chang, P., \& Broderick, A. E. 2012, ApJ, 752, 24

Puchwein, E., Pfrommer, C., Springel, V., Broderick, A. E., \& Chang, P. 2012, MNRAS, 423, 149

Schlickeiser, R., Ibscher, D., \& Supsar, M. 2012, ApJ, 758, 102

Schlickeiser, R., Krakau, S., \& Supsar, M. 2013, ApJ, 777, 49

Shalaby, M., Broderick, A. E., Chang, P., et al. 2017a, ApJ, 848, 81 Shalaby, M., Broderick, A. E., Chang, P., et al. 2017b, ApJ, 841, 52 Thurgood, J. O., \& Tsiklauri, D. 2016, JPIPh, 82, 905820604

Tiede, P., Broderick, A. E., Shalaby, M., et al. 2017a, ApJ, 850, 157

Tiede, P., Broderick, A. E., Shalaby, M., et al. 2017b, arXiv:1702.02586

Timofeev, I. V., Lotov, K. V., \& Terekhov, A. V. 2009, PhPl, 16, 063101

Vafin, S., Rafighi, I., Pohl, M., \& Niemiec, J. 2018, ApJ, 857, 43

Voshchepynets, A., \& Krasnoselskikh, V. 2013, AnGeo, 31, 1379

Voshchepynets, A., Krasnoselskikh, V., Artemyev, A., \& Volokitin, A. 2015, ApJ, 807, 38 